\title{
Embracing Computational Approaches Can Stimulate Clinical Psychology Research
}

\author{
Omer Van den Bergh ${ }^{\mathrm{a}}$, Nadine Lehnen ${ }^{\mathrm{b}}$
}

[a] Health Psychology, University of Leuven, Leuven, Belgium. [b] Department of Psychosomatic Medicine and Psychotherapy, Technical University of Munich, Munich, Germany.

Clinical Psychology in Europe, 2019, Vol. 1(3), Article e39237, https://doi.org/10.32872/cpe.v1i3.39237

Published (VoR): 2019-09-20

Corresponding Author: Omer Van den Bergh, University of Leuven, Health Psychology, Tiensestraat 102, 3000 Leuven, Belgium. E-mail: omer.vandenbergh@kuleuven.be

Clinical psychology is predominantly a "verbal" science: we derive most clinically useful information from what people say and talking is a critical means in the preferred unit of intervention: person-to-person interaction. Psychologists often tend to believe that numbers are a poor means of capturing and representing what goes on in the individual's mind and sometimes consider attempts to do so as naïve, if not offensive, to the essence of human nature and existence. One of the arguments advanced cites "complexity": the human mind is simply too rich and complex to reduce it to numbers. Interestingly, complexity in other sciences and clinical specialties is often cited as one of the main reasons to use computing and to develop mathematical models and apply simulations. Should clinical psychology consider going down this path?

As a scientific endeavor, clinical psychology is (and should be) rooted in empirical data and validated theoretical models that allow prediction. Indeed, in a broad sense, both diagnostic and therapeutic steps (implicitly) involve a probabilistic prediction about future behavior. One way to validate models is to carry out experiments. However, reality in experiments is artificially reduced and controlled in order to test the effect of one or only a small set of independent (manipulated) variables on some variable of interest. The benefit is that they allow us to detect causal relationships and develop heuristics to understand behavior. This is why experiments should be simple: our human mind can hardly grasp a 2-way interaction, let alone a 3- or 4-way interaction ${ }^{1}$. However, since multiple higher order interactions and recursive effects (effects feed back on causes) are the rule in life, experiments do not allow us to predict actual behavior in a real context.

1) Courtesy for this statement to my old professor of statistics, OVdB 
This is no different to natural sciences: Just like experimentally investigating the relationship between pressure, temperature and volume of a gas is important to eventually understand weather systems, the equations generated in experiments will not enable us to predict the weather across the next few days. The latter implies more complicated computational models with deterministic and stochastic variables in which lab-based equations act as building blocks that are fed with initial data and that are continuously updated as new information unfolds. Eventually, our human mind may not be able to fully grasp all the higher-order interactions, but nevertheless we may become quite good at predicting the weather.

Computational science as an interdisciplinary field develops concepts, methods and tools to mathematically model and analyze complex problems and systems. It is, by itself, rather content-free. Computational approaches have been successfully used in neurosciences for a long time (Sejnowski, Koch, \& Churchland, 1988; Huys, Maia, \& Frank, 2016) and have been promoted in psychiatry (Friston, Stephan, Montague, \& Dolan, 2014; Petzschner, 2017) and psychosomatics (Petzschner, Weber, Gard, \& Stephan, 2017). Computational approaches are advocated, for example, to bridge the gap between neural pattern activity and behavioral data (Stephan \& Mathys, 2014), to improve (data-driven) phenotyping of patients (Patzelt, Hartley, \& Gershman, 2018), and to develop, test and improve theoretical explanatory models through simulation (Lehnen et al., submitted). A recent first attempt at the latter approach, combining mathematical formulization of an existing explanatory model with experiments, has proven useful to deepen our understanding of the complex mechanisms underlying persistent physical symptoms (Lehnen, Schröder, Henningsen, Glasauer, \& Ramaioli, 2019).

How relevant is this for clinical psychology in practice? Several important new developments will probably force us to go in this direction. First, ecological momentary assessments will undoubtedly become increasingly standard to measure self-reported variables of cognitive and affective processes and social interactions while they are occurring. Second, it will increasingly become standard to concurrently collect psychophysiological and behavioral data through unobtrusive body sensors. Both sources of information in real life will generate large multilevel sets of data per person in multiple conditions. Since clinical psychology is primarily concerned with care for an individual patient in a particular context, this is exactly the kind of data that is relevant for personalized care. Individualized functions comprising deterministic and stochastic variables that model observations registered across multiple occasions in multiple relevant contexts actually represent a theory of an individual that may act as an empirically based tool to expect/predict (and understand) behavior. In addition, such functions can be used to assess step by step change over time in a therapeutic process. Aggregation of single-case data may enable us to generalize and develop data-driven models and theories and/or to test and refine existing theoretical models. Such an approach, which has already been successfully applied in other clinical specialties (for a recent example see Glasauer, Dieterich, \& Brandt, 2018), 
turns the current situation upside down: rather than using heuristics that are based on experiments to intuitively predict/understand behavior of an individual patients in a particular context, the reverse sequence might result in quite different models that, for example, attribute much more weight to contextual variables. Obviously, this may require clinical psychologists to be trained in a completely different way, as well as may require much more interdisciplinary collaboration.

\section{References}

Friston, K. J., Stephan, K. E., Montague, R., \& Dolan, R. J. (2014). Computational psychiatry: The brain as a phantastic organ. Lancet Psychiatry, 1(2), 148-158.

https://doi.org/10.1016/S2215-0366(14)70275-5

Glasauer, S., Dieterich, M., \& Brandt, T. (2018). Neuronal network-based mathematical modeling of perceived verticality in acute unilateral vestibular lesions: From nerve to thalamus and cortex. Journal of Neurology, 265(Suppl 1), 101-112. https://doi.org/10.1007/s00415-018-8909-5

Huys, Q. J., Maia, T. V., \& Frank, M. J. (2016). Computational psychiatry as a bridge from neuroscience to clinical applications. Nature Neuroscience, 19(3), 404-413. https://doi.org/10.1038/nn.4238

Lehnen, N., Radziej, K., Weigel, A., von Känel, R., Glasauer, S., Pitron, V., ... Henningsen, P. on behalf of the EURONET-SOMA Group (submitted). Computational-experimental psychosomatics for a better understanding of persistent somatic symptoms. Manuscript submitted for publication.

Lehnen, N., Schröder, L., Henningsen, P., Glasauer, S., \& Ramaioli, C. (2019). Deficient head motor control in functional dizziness: Experimental evidence of central sensory-motor dysfunction in persistent physical symptoms. Progress in Brain Research, 249, 385-400. https://doi.org/10.1016/bs.pbr.2019.02.006

Patzelt, E. H., Hartley, C. A., \& Gershman, S. J. (2018). Computational phenotyping: Using models to understand individual differences in personality, development, and mental illness. Personality Neuroscience, 1, Article e18. https://doi.org/10.1017/pen.2018.14

Petzschner, F. H. (2017). Stochastic dynamic models for computational psychiatry and computational neurology. Biological Psychiatry: Cognitive Neuroscience and Neuroimaging, 2(3), 214-215. https://doi.org/10.1016/j.bpsc.2017.03.003

Petzschner, F. H., Weber, L. A., Gard, T., \& Stephan, K. E. (2017). Computational psychosomatics and computational psychiatry: Toward a joint framework for differential diagnosis. Biological Psychiatry, 82(6), 421-430. https://doi.org/10.1016/j.biopsych.2017.05.012

Sejnowski, T. J., Koch, C., \& Churchland, P. S. (1988). Computational neuroscience. Science, 241(4871), 1299-306. https://doi.org/10.1126/science.3045969

Stephan, K. E., \& Mathys, C. (2014). Computational approaches to psychiatry. Current Opinion in Neurobiology, 25, 85-92. https://doi.org/10.1016/j.conb.2013.12.007 


\section{EACLIPT}

Clinical Psychology in Europe (CPE) is the official journal of the European Association of Clinical Psychology and Psychological Treatment (EACLIPT).

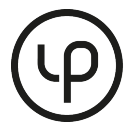

leibniz-psychology.org

PsychOpen GOLD is a publishing service by Leibniz Institute for Psychology Information (ZPID), Germany. 